Case Report

\title{
Addisonian Crisis due to Metastatic Adenocarcinoma in a Pygmy Goat
}

\author{
Nora Nogradi, ${ }^{1}$ Amanda L. Koehne, ${ }^{1}$ F. Charles Mohr, ${ }^{2}$ \\ Sean D. Owens, ${ }^{2}$ and Meera C. Heller ${ }^{3,4}$ \\ ${ }^{1}$ William R. Pritchard Veterinary Medical Teaching Hospital, University of California, Davis, One Shield Avenue, Davis, \\ CA 95616, USA \\ ${ }^{2}$ Department of Pathology, Microbiology and Immunology, School of Veterinary Medicine, University of California Davis, \\ Davis, One Shield Avenue, Davis, CA 95616, USA \\ ${ }^{3}$ Department of Medicine and Epidemiology, School of Veterinary Medicine, University of California Davis, Davis, \\ One Shield Avenue, Davis, CA 95616, USA \\ ${ }^{4}$ Department of Veterinary Medicine and Surgery, University of Missouri, 900 East Campus Drive, Columbia, MO 65211, USA
}

Correspondence should be addressed to Meera C. Heller; hellerme@missouri.edu

Received 31 May 2013; Accepted 18 July 2013

Academic Editors: N. D. Giadinis, C. Hyun, L. G. Papazoglou, and M. Pizarro

Copyright (C) 2013 Nora Nogradi et al. This is an open access article distributed under the Creative Commons Attribution License, which permits unrestricted use, distribution, and reproduction in any medium, provided the original work is properly cited.

\begin{abstract}
A 15-year-old Pygmy doe was evaluated for acute onset of lethargy, anorexia, and weakness. Adrenal insufficiency was diagnosed based on physical exam findings, blood work abnormalities (hyponatremia, hyperkalemia, azotemia, and hypoglycemia), and lack of cortisol response to the ACTH stimulation test. Abdominal ultrasound exam revealed an intact urinary tract and multiple bilateral peri-renal masses. The doe was treated with intravenous fluid therapy aimed at correcting the electrolyte abnormalities and intravenous corticosteroids. She responded favorably to medical therapy in 24 hours, with dramatic improvement in attitude and appetite. Fluid therapy was discontinued, and the doe was discharged from the hospital on steroid supplementation. She deteriorated rapidly and died at home 36 hours after discharge. Necropsy results revealed metastatic adenocarcinoma originating from the uterus that infiltrated the urinary bladder, the region of the adrenal glands, the left and right renal lymph nodes, the left kidney, the caudal vena cava, the submandibular lymph nodes, the diaphragm, the lungs, and the omentum. Addison's syndrome in ruminants should be considered as an uncommon sequel of intra-abdominal neoplastic processes.
\end{abstract}

\section{Introduction}

To the authors' knowledge, this is the first clinical report of Addison's syndrome in a goat. Addison's syndrome describes a clinical phenomenon when the adrenal cortex is unable to produce steroid hormones, in particular the corticosteroid cortisol and the mineralocorticoid aldosterone. Cortisol is produced by the zona fasciculata of the adrenal cortex, and its primary functions are in the maintenance of blood pressure, cardiovascular function, and physiologic glucose levels as well as the regulation of protein, carbohydrate, and fat metabolism. Aldosterone is produced by the zona glomerulosa of the adrenal cortex and acts on the collecting ducts and the distal convoluted tubules in the kidneys. It is responsible for conservation of sodium, water retention, maintenance of blood pressure, and secretion of potassium [1].
Addison's disease is a well-described clinical syndrome in dogs, characterized by diarrhea, vomiting, lethargy, anorexia, muscle weakness, hyperkalemia, hyponatremia, hypoglycemia, and lack of corticosteroid-induced stress leukogram despite illness [2]. The doe in this case report presented with similar, nonspecific clinical signs and, along with the characteristic serum electrolyte and biochemical abnormalities, raised the clinician's suspicion for primary hypoadrenocorticism.

\section{Case Presentation}

A 15-year-old Pygmy doe was presented to the Veterinary Medical Teaching Hospital of the University of California, Davis, for an acute onset of anorexia, lethargy, and weakness 
of 2-day duration that progressed into recumbency on the evening of evaluation. The doe was individually housed in a barn, had access to a grass pasture, and was supplemented with wheat hay. At the time of presentation, she was not current on routine vaccinations, had not recently been treated for endo- or ectoparasites, and had not received any medical treatment, including pharmacologic agents, prior to presentation. At presentation, she weighed $19 \mathrm{~kg}$ and had a body condition score of 2 out of 5 . She was obtunded and exhibited profound weakness. On physical examination, a normal rectal temperature and respiratory rate were noted, and mild tachycardia $(100 \mathrm{bpm})$ was observed. Mucous membranes were pale and tacky with a capillary refill time of 3 seconds. Skin turgor was delayed, and peripheral pulse quality was weak. Eyes, ears, and nose were clean; lice infestation of the skin was evident. No murmurs or arrhythmias were evident on cardiac auscultation; normal bronchovesicular sounds were auscultated in all lung fields bilaterally. No ruminal contractions could be auscultated; however, the doe was observed to pass feces at the time of presentation, and a fecal occult blood test was negative. A brief neurologic exam revealed obtundation, intact cranial nerves and spinal reflexes, and profound muscle weakness, and the doe was unable to stand with assistance. Abnormalities on blood work available after hours included anemia (17\%, ref.: $23-36 \%)$ with normal total protein concentration $(7 \mathrm{~g} / \mathrm{dL}$; ref.: $6.8-8.3 \mathrm{~g} / \mathrm{dL})$, hyponatremia ( $132 \mathrm{mmol} / \mathrm{L}$; ref.: $140-150 \mathrm{mmol} / \mathrm{L})$, hyperkalemia (6.8 mmol/L; ref.: $3.4-5.7 \mathrm{mmol} / \mathrm{L})$, hypoglycemia (<20 mg/dL; ref.: $45-80 \mathrm{mg} / \mathrm{dL}$ ), and azotemia (creatinine: $2.4 \mathrm{mg} / \mathrm{dL}$; ref.: $0.7-1 \mathrm{mg} / \mathrm{dL}$ ). A urinalysis was not performed as urine could not be obtained at the time of presentation. Abdominal ultrasound was performed, and free abdominal fluid was not observed. The urinary bladder was found to be intact, and bilateral renomegaly with abnormal renal echogenicity and thickened renal cortices $(1 \mathrm{~cm})$ was noted. Multiple diffusely hyperechoic masses were observed in the inguinal and perirenal regions (Figure 1). The uterus, containing hypoechoic fluid, was identified in the right inguinal region, and a wall thickness of $5 \mathrm{~mm}$ was observed. Small intestinal bowel loops were visualized in the ventral abdomen. Decreased peristaltic activity and increased wall thickness $(4 \mathrm{~mm})$ were noted.

The doe was started on replacement fluid therapy with isotonic sodium-chloride solution at a dose of $20 \mathrm{~mL} / \mathrm{kg}$ in order to treat the electrolyte abnormalities, with 5\% dextrose added to the fluids to treat the severe hypoglycemia. Attitude and vital parameters improved after the fluid bolus, blood glucose levels normalized, and the doe was maintained on isotonic sodium-chloride supplemented with $2 \%$ dextrose at a rate of $4 \mathrm{~mL} / \mathrm{kg} / \mathrm{hr}$ overnight to promote diuresis and ameliorate the azotemia. The doe's attitude slightly improved on day 2: she stood on her own and was picking at food; she was passing feces and urinating normally. Complete blood count revealed normocytic, normochromic anemia with increased reticulocyte count suggestive of a regenerative process. The leukogram was unremarkable, and total protein and fibrinogen concentrations were within normal limits. Serum biochemistry panel on day 2 revealed improvement in

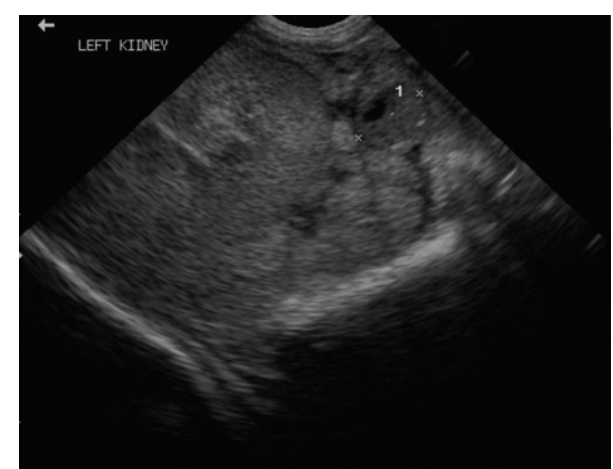

FIGURE 1: Ultrasonographic image of the left kidney obtained from the right paralumbar fossa. Note the increased thickness of the renal cortex and the multiple masses in the perirenal region. Obtained with a $5 \mathrm{MHz}$ microconvex transducer. Left is dorsal; right is ventral.

the hyponatremia (139 mmol/L; ref.: $140-150 \mathrm{mmol} / \mathrm{L})$, normal glucose concentration ( $75 \mathrm{mg} / \mathrm{dL}$; ref.: $45-80 \mathrm{mg} / \mathrm{dL}$ ) but no significant change in the azotemia (creatinine: $2.4 \mathrm{mg} / \mathrm{dL}$; ref.: $0.7-1 \mathrm{mg} / \mathrm{dL}$, BUN: $104 \mathrm{mg} / \mathrm{dL}$; ref.: $19-31 \mathrm{mg} / \mathrm{dL}$ ) and hyperkalemia $(6.7 \mathrm{mmol} / \mathrm{L}$; ref.: $3.4-5.7 \mathrm{mmol} / \mathrm{L})$. The doe was also hypercalcemic (11.6 mg/dL; ref.: $8.9-11.2 \mathrm{mg} / \mathrm{dL})$ and had a decreased albumin concentration $(2.7 \mathrm{~g} / \mathrm{dL}$; ref.: $3.8-$ $4.5 \mathrm{~g} / \mathrm{dL}$ ). Urinalysis revealed isosthenuria (USG: 1.010 ) and no other abnormal findings. An abdominal ultrasound exam was repeated which was found to be consistent with the findings from the previous day. The problem list in this doe included low body condition score, fatigue, obtundation, anemia, azotemia, hyperkalemia, hyponatremia, hypercalcemia, hypoalbuminemia, and perirenal masses detected on the abdominal ultrasound examination.

This doe had no history of prolonged anorexia, and her daily feed determined to be of good quality. As such, a systemic disease process or endoparasitism was considered the most likely differentials for her decreased body condition score. Her acute onset of fatigue and obtundation was thought to be due to the severe hypoglycemia, as her mentation and strength improved after dextrose supplementation. Hypoglycemia in an adult animal may result from excessive glucose utilization, neoplasia (insulinoma), impaired hepatic gluconeogenesis and glycogenolysis (hepatic insufficiency), diabetogenic hormone (cortisol) deficiency, sepsis, or a combination of these mechanisms [3]. This doe had normal liver enzymes and no evidence of sepsis based on the physical exam and the leukogram, so increased glucose uptake by the cells, hyperinsulinemia due to insulin producing tumor, and cortisol deficiency were considered likely causes of her severe hypoglycemia. This patient's azotemia and severe electrolyte disturbances in light of normal or adequate urine production were suggestive of polyuric renal failure or hypoadrenocorticism. Unfortunately, a urine specific gravity was not obtained prior to initiation of fluid therapy. However, the persistently elevated creatinine in the face of fluid therapy and diuresis was suggestive of impaired renal function. Although hyperkalemia is often observed with oliguric renal failure, the magnitude of the hyperkalemia in this patient and the 
lack of response to intravenous fluid therapy and glucose supplementation in addition to the severe hypoglycemia and fatigue were suggestive of adrenal insufficiency. Further, the presence of regenerative anemia, in concert with the low serum albumin concentration, and the absence of evidence to support hemolysis suggested blood loss. As the doe had no history of external hemorrhage and fecal occult blood test was negative, the ectoparasitism detected on the physical exam was considered the most likely cause of the anemia and hypoalbuminemia. The observed hypercalcemia in this patient is an uncommon finding in ruminants and is usually associated with hypervitaminosis $\mathrm{D}$ due to iatrogenic or toxic causes [4]. Additionally, elevated total calcium levels in ruminants can also be associated with pseudohyperparathyroidism due to a neoplastic process, and hypercalcemia can also be associated with hypoadrenocorticism in other species [5].

Following the initial diagnostic tests, additional diagnostic tests performed included fecal egg count determination (which was negative) and performance of an ACTH (adrenocorticotropic hormone) stimulation test to evaluate the function of the adrenal cortex. Baseline cortisol concentrations were undetectable, and thus a follow-up ACTH stimulation test was performed using a dosage of synthetic ACTH of $10 \mu \mathrm{g} / \mathrm{kg}$. This dose was extrapolated from the results of a previous report [6]. Serum cortisol concentrations 1 hour after ACTH administration remained below detection range levels, and the diagnosis of adrenal insufficiency was made. An intra-abdominal neoplastic process was considered most likely with direct involvement of the adrenal glands. A biopsy of the peri-renal masses and the kidneys was not performed due to lack of owner consent. As the owner elected for supportive care only, the doe was treated with a single dose of prednisolone sodium succinate intravenously $(2 \mathrm{mg} / \mathrm{kg})$ to address the glucocorticoid deficiency and then maintained on once daily intravenous dexamethasone $(0.2 \mathrm{mg} / \mathrm{kg})$. The severe hyperkalemia was a concern, but unfortunately, mineralocorticoid administration was not an option in this doe due to financial constraints. She was given low dose furosemide intravenously $(0.25 \mathrm{mg} / \mathrm{kg})$ once to increase urinary excretion of potassium, while maintenance fluid therapy with dextrose supplementation was continued. The doe was significantly brighter and stronger on day 3; she had normal vital parameters and rumen contractions, maintained a good appetite, and was passing manure and urine normally. Blood work revealed resolution of the electrolyte abnormalities and persistent azotemia, therefore fluid therapy was continued with Normosol-R. She was able to maintain physiologic glucose levels with voluntary feeding and the steroid therapy, and she was subsequently weaned off dextrose supplementation. Glucose and electrolyte concentrations were normal on day 4 , while the azotemia was unchanged (creatinine: $2.3 \mathrm{mg} / \mathrm{dL}$; ref.: $0.7-1 \mathrm{mg} / \mathrm{dL}$; BUN: $123 \mathrm{mg} / \mathrm{dL}$; ref.: $19-31 \mathrm{mg} / \mathrm{dL}$ ), and the diagnosis of chronic renal insufficiency was made. She was tapered off the intravenous fluid therapy and given a dose of long acting methylprednisolone acetate $(1 \mathrm{mg} / \mathrm{kg}$ intramuscular) in order to prepare her for discharge on day 5. The owner reported signs of abdominal discomfort and restlessness the day following discharge and brought the doe back to the hospital 36 hours after discharge, but she was deceased on arrival. A full necropsy revealed widespread neoplasia, which was histologically confirmed as adenocarcinoma. The largest and presumably primary mass was in the uterus with infiltration into the lungs, diaphragm, left kidney, urinary bladder, omentum, sublumbar, and left and right renal lymph nodes and to the region of the adrenal glands where adrenal tissue could not be identified grossly or histologically. Adjacent to the right kidney, the neoplasm extended into the caudal vena cava and occluded the lumen. In addition, a thrombus was attached to the intravascular tumor (Figure 2). Effacement of the adrenal glands by the neoplasm presumably led to the doe's clinical presentation consistent with hypoadrenocorticism. The acute onset of abdominal pain and rapid deterioration of the doe may have been due to the occluding tumor metastasis and thrombus in the caudal vena cava.

\section{Discussion}

Primary hypoadrenocorticism has been previously reported in South African angora goats, resulting in increased sensitivity of these animals to weather changes [6], which appears to be genetic in this breed [7]. Diagnosis of Addison's syndrome is based on results of the ACTH stimulation test [3]. As this test is not routinely performed in goats, the ACTH dose used in this doe was selected based on a previous study, where it was successfully used to evaluate adrenal function in healthy Angora and Boer goats [6]. Another experimental model successfully used both the low dose $(0.4 \mathrm{IU} / \mathrm{kg}=4 \mu \mathrm{g} / \mathrm{kg})$ and high dose $(2.5 \mathrm{IU} / \mathrm{kg}=25 \mu \mathrm{g} / \mathrm{kg})$ ACTH stimulation tests in Angora and Spanish wethers [7]. Physiologic resting cortisol concentrations have been reported to be between 1 and $15 \mathrm{ng} / \mathrm{mL}$ in healthy, adult goats [6-8], and a normal response was considered to be a minimum of 3-4-fold elevation of plasma cortisol after ACTH administration [6, 7]. In dogs, a standard dose of synthetic ACTH $(250 \mu \mathrm{g} / \mathrm{dog})$ is used for evaluation of adrenocortical function, regardless of the size of the dog, and a normal response is considered to be an 8-10fold elevation in the cortisol concentration when compared to resting levels [9]. However, a validated testing protocol has not been described in goats with primary hypoadrenocorticism. Based on previous research $[6,7]$ and our findings in this case, we conclude that the ACTH stimulation protocol used in this goat proved to be a reliable way to diagnose Addison's syndrome.

Addison's syndrome most commonly occurs in young, female dogs and is due to an immune mediated process destructing the adrenal cortices [10]. There is one case report of a 12-year-old dog diagnosed with Addison's syndrome due to bilateral adrenal neoplasia [11]. That dog presented with clinical signs and laboratory alterations consistent with Addison's disease but had an uncharacteristic signalment and bilaterally enlarged adrenal glands on the abdominal ultrasound exam. The dog was started on standard replacement therapy with dexamethasone and fludrocortisone, and after an initial positive response to therapy, it acutely deteriorated in 5 days showing signs of restlessness and abdominal 


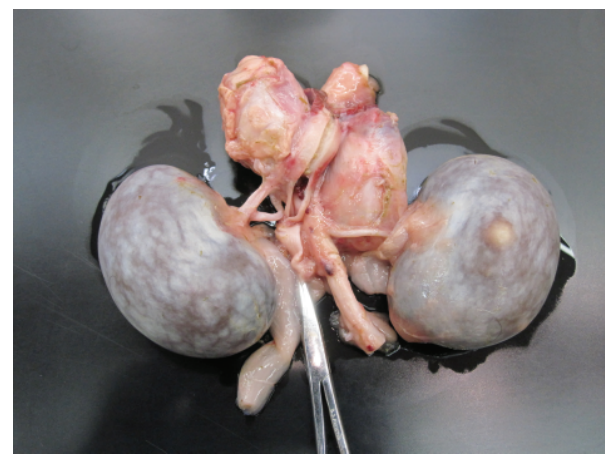

FIGURE 2: Gross image of kidneys and tumor masses. Tumor nodules are present between the kidneys in the region of the adrenal glands and renal lymph nodes and also within the caudal vena cave (asterisk). The left kidney has a small tumor nodule underneath the capsule. Top: cranial, bottom: caudal. The ruler spans $2 \mathrm{~cm}$.

discomfort. Histological examination of the adrenal glands after euthanasia revealed highly anaplastic bilateral adrenal neoplasia. According to a recent retrospective study, the average rate of metastatic involvement of the adrenal glands is $21 \%$ in dogs, $26.9 \%$ in horses, and $31.3 \%$ in cattle [12]. No goat necropsies were involved in that study. Common tumor types that metastasize to the adrenal gland include carcinoma, adenocarcinoma, and lymphoma. Only one dog was reported to have developed Addison's disease secondary to the metastatic neoplasia [12]. Necropsy findings in this doe revealed that the metastatic adenocarcinoma originated from the uterus and infiltrated multiple abdominal organs. There are a couple of single case reports of uterine neoplasia of muscle origin in goats $[13,14]$ and one report of metastasized adenocarcinoma of the uterus [15], but no case series of uterine neoplasia are described. Based on studies in other species [16], we may assume that they are more common in the geriatric population.

Dogs diagnosed with Addison's syndrome often have azotemia, which is considered to be prerenal in origin and due to the dehydration [10]. The azotemia in this doe was unresponsive to diuresis, suggestive of renal origin. Dogs diagnosed with primary hypoadrenocorticism require lifelong replacement therapy with corticosteroids and mineralocorticoids and are reported to maintain a good quality of life [17]. Treatment of the doe in this case report was aimed at correcting the severe electrolyte abnormalities with intravenous fluid therapy and corticosteroid supplementation. As mineralocorticoid administration was not an option due to financial constraints, she was given a single dose of furosemide to increase urinary excretion of potassium. Such treatment is controversial in animals with hypoadrenocorticism, due to the risk of exacerbating the hypotension, although, a partial, single dose with concurrent administration of intravenous fluids was considered to be a safe alternative to mineralocorticoid supplementation in this case. While dexamethasone has no activity on the mineralocorticoid receptors, methylprednisolone and prednisolone both have significant effects on this receptor type [18]. No previous reports described treatment of Addison's disease with corticosteroids only; it was attempted in this doe as the sole option to lengthen the animal's life. As the doe deteriorated shortly after discharge, while the long acting corticosteroids were still supposed to have adequate circulating levels, inadequate stress response was not considered to be the primary cause of her death but could have contributed to the pain and discomfort of this animal because of its role in promoting a hypercoagulable state [19] leading to thrombosis of the caudal vena cava.

In conclusion, Addison's syndrome as sequelae to neoplasia can occur in goats, and an ACTH stimulation test using synthetic ACTH intravenously can be used to diagnose the disease. As no other causes of primary hypoadrenocorticism have been previously reported in goats, consideration of an intra-abdominal neoplastic process is recommended. The success of replacement therapy likely depends on the initiating cause and potential involvement of organs other than the adrenal glands.

\section{References}

[1] S. R. Bornstein, "Predisposing factors for adrenal insufficiency," The New England Journal of Medicine, vol. 360, no. 22, pp. 23282339, 2009.

[2] S. C. Klein and M. E. Peterson, "Canine hypoadrenocorticism: part I," Canadian Veterinary Journal, vol. 51, no. 1, pp. 63-69, 2010.

[3] R. W. Nelson, G. H. Turnwald, and M. D. Willard, "Endocrine, metabolic, and lipid disorders," in Small Animal Clinical Diagnosis By Laboratory Methods, M. D. Willard and T. Harold, Eds., pp. 165-207, Elsevier, St. Louis, Mo, USA, 2004.

[4] G. P. Carlson, "Clinical chemistry tests," in Large Animal Internal Medicine, B. P. Smith, Ed., pp. 375-397, Mosby, St. Louis, Mo, USA, 2009.

[5] P. A. Schenck, D. J. Chew, L. A. Nagode, T. J. Rosol et al., "Disorders of calcium: hypercalcemia and hypocalcemia," in Fluid, Electrolyte, and Acid-Base Disorders in Small Animal Practice, S. P. DiBartola, Ed., Elsevier, St. Louis, Mo, USA, 3rd edition, 2006.

[6] Y. Engelbrecht, T. Herselman, A. Louw, and P. Swart, "Investigation of the primary cause of hypoadrenocorticism in South African Angora goats (Capra aegagrus): a comparison with Boer goats (Capra hircus) and Merino sheep (Ovis aries)," Journal of Animal Science, vol. 78, no. 2, pp. 371-379, 2000.

[7] C. A. Toerien, R. Puchala, J. P. McCann, T. Sahlu, and A. L. Goetsch, "Adrenocortical response to ACTH in Angora and Spanish goat wethers," Journal of Animal Science, vol. 77, no. 6, pp. 1558-1564, 1999.

[8] L. Eriksson and T. L. Teravainen, "Circadian rhythm of plasma cortisol and blood glucose in goats," Australian Journal of Applied Science, pp. 202-203, 1989.

[9] B. P. Meij and J. A. Mol, "Adrenocortical function," in Clinical Biochemistry of Domestic Animals, J. J. Kaneko, J. W. Harvey, and M. Bruss, Eds., pp. 605-622, Elsevier, St. Louis, Mo, USA, 2008.

[10] R. W. Nelson, "Disorders of the adrenal gland," in Small Animal Internal Medicine, R. W. Nelson and C. G. Couto, Eds., pp. 778815, Mosby, St. Louis, Mo, USA, 2003.

[11] P. H. Kook, P. Grest, U. Raute-Kreinsen, C. Leo, and C. E. Reusch, "Addison's disease due to bilateral adrenal malignancy in a dog," Journal of Small Animal Practice, vol. 51, no. 6, pp. $333-336,2010$. 
[12] P. Labelle and H. E. V. De Cock, "Metastatic tumors to the adrenal glands in domestic animals," Veterinary Pathology, vol. 42, no. 1, pp. 52-58, 2005.

[13] F. A. Uzal and B. Puschner, "Cervical leiomyoma in an aged goat leading to massive hemorrhage and death," Canadian Veterinary Journal, vol. 49, no. 2, pp. 177-179, 2008.

[14] K. M. Whitney, B. A. Valentine, and D. H. Schlafer, "Caprine genital Leiomyosarcoma," Veterinary Pathology, vol. 37, no. 1, pp. 89-94, 2000.

[15] P. Pfister, U. Geissbuehler, D. Wiener, G. Hirsbrunner, and C. Kaufmann, "Pollakisuria in a dwarf goat due to pathologic enlargement of the uterus," Veterinary Quarterly, vol. 29, no. 3, pp. 112-116, 2007.

[16] S. J. Woodhouse and C. S. Hanley, "What is your diagnosis? Uterine adenocarcinoma," Journal of the American Veterinary Medical Association, vol. 238, no. 3, pp. 289-290, 2011.

[17] S. C. Klein and M. E. Peterson, "Canine hypoadrenocorticism: part II," Canadian Veterinary Journal, vol. 51, no. 2, pp. 179-184, 2010.

[18] M. J. Day, "Glucocorticoids and antihistamines," in Small Animal Clinical Pharmacology, J. E. Maddison, S. W. Page, and D. B. Church, Eds., pp. 261-269, Elsevier, 2008.

[19] M. A. Kerachian, D. Cournoyer, E. J. Harvey et al., "Effect of high-dose dexamethasone on endothelial haemostatic gene expression and neutrophil adhesion," Journal of Steroid Biochemistry and Molecular Biology, vol. 116, no. 3-5, pp. 127-133, 2009. 

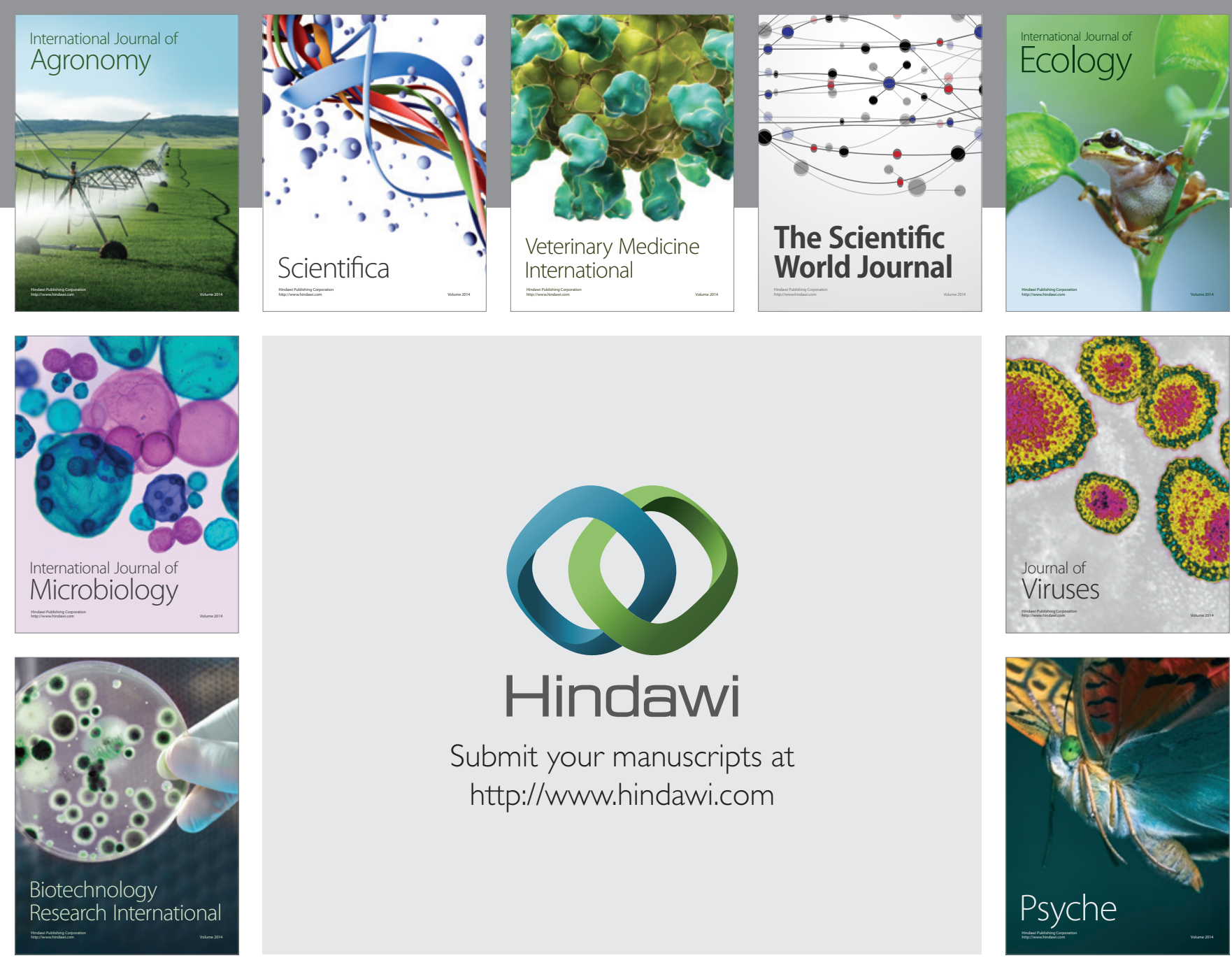

Submit your manuscripts at http://www.hindawi.com
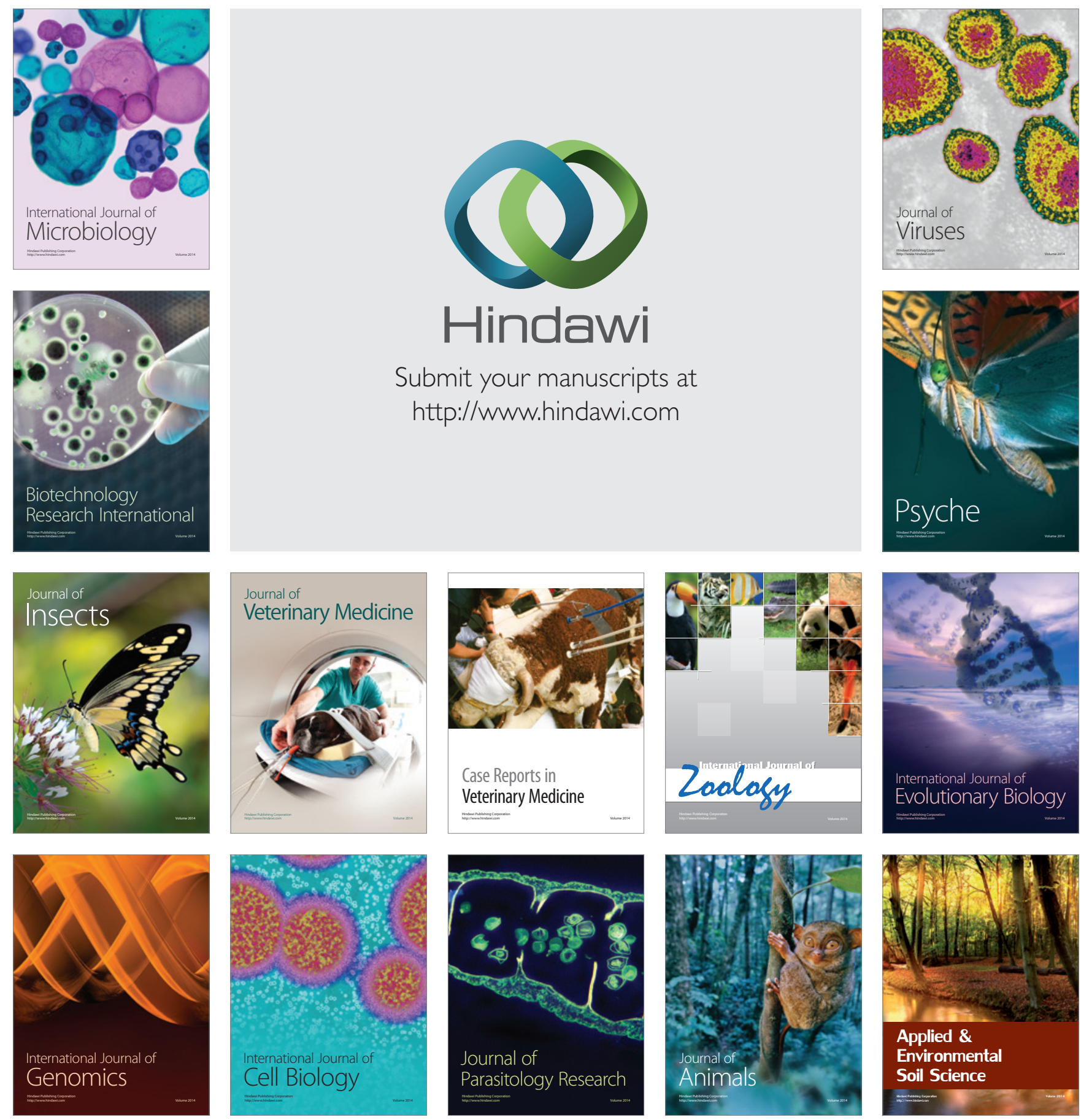